\title{
Degenerative Pannus Mimicking Clival Chordoma Resected via an Endoscopic Transnasal Approach
}

\author{
Ahmad Khaldi, M.D., ${ }^{1}$ Julius Griauzde, B.A., ${ }^{1}$ and Edward A.M. Duckworth, M.D. ${ }^{2}$
}

Lesions of the lower clivus represent a technically challenging subset of skull base disease that requires careful treatment. A 75-year-old woman with tongue atrophy was referred for resection of a presumed clival chordoma. The lesion was resected via an endoscopic transnasal transclival approach with no complications. Pathology revealed only chronic inflammatory tissue consistent with a degenerative pannus. Degenerative pannus should be included in the differential diagnosis of lower clival extradural lesions. The endoscopic transnasal transclival corridor should be considered for resection of such lesions as an alternative to larger, more morbid, traditional skull base approaches.

KEYWORDS: Clivus, endoscopic, chordoma, pannus, degenerative

The clivus is comprised of the continuum of the dorsum sella of the sphenoid bone rostrally and the basal portion of the occipital bone caudally. ${ }^{1}$ The anatomic location of the clivus makes surgery for skull base lesions challenging. Adjacent vital structures include the internal carotid artery, optic nerves, cavernous sinus, brain stem, and lower cranial nerves. Although access is difficult, surgery of the clivus can be indicated in an attempt to cure, for diagnosis, or to decompress mass effect.

Primary lesions found in this region include chordomas, meningiomas, pituitary adenomas, fibrous dysplasia, and mucoceles. Metastatic lesions also occur and include lymphomas, nasopharyngeal carcinoma, and squamous cell carcinomas. ${ }^{2}$ In the past, computed tomography $(\mathrm{CT})$-guided biopsy has been utilized for diagnosis, with possible treatment coming in the form of traditional open skull base surgical approaches. More recently, endo- scopic techniques have been used for clival pathology with minimal morbidity. ${ }^{2-5}$ The evolution of endoscopic approaches has resulted from collaboration between otolaryngologists and neurosurgeons, as well as from technical advances such as the development of more sophisticated neuronavigation platforms, extendable microinstruments, and improved endoscopic optics. ${ }^{6}$

Rheumatoid disease is characterized by an autoimmune reaction against synovial joint cells, with the release of a complex milieu of inflammatory mediators. ${ }^{7}$ The corresponding cascade of cellular interactions results in the recruitment and activation of fibroblasts and formation of granulation tissue, which, when advanced, can form into a rheumatoid pannus. ${ }^{8}$ Rheumatoid disease with pannus formation is commonly found at the craniovertebral junction, ${ }^{8}$ or often retro-odontoid. ${ }^{9}$

In the present report, we describe the case of a 75-year-old woman found to have a mass involving the
${ }^{1}$ Departments of Neurosurgery at Loyola University Stritch School of Medicine, Maywood, Illinois; ${ }^{2}$ Baylor College of Medicine, Houston, Texas.

Address for correspondence and reprint requests: Edward A.M. Duckworth, M.D., Assistant Professor, Director of Cerebrovascular and Skull Base Neurosurgery, Department of Neurosurgery, Baylor College of Medicine, 1709 Dryden Road, Suite 750, Houston, TX 77030 (e-mail: edward.duckworth@bcm.edu).
Skull Base Rep 2011;1:7-12. Copyright (C) 2011 by Thieme Medical Publishers, Inc., 333 Seventh Avenue, New York, NY 10001, USA. Tel: +1(212) 584-4662.

Received: March 10, 2010. Accepted after revision: August 3, 2010. Published online: March 30, 2011.

DOI: http://dx.doi.org/10.1055/s-0031-1275243.

ISSN 2157-6971. 

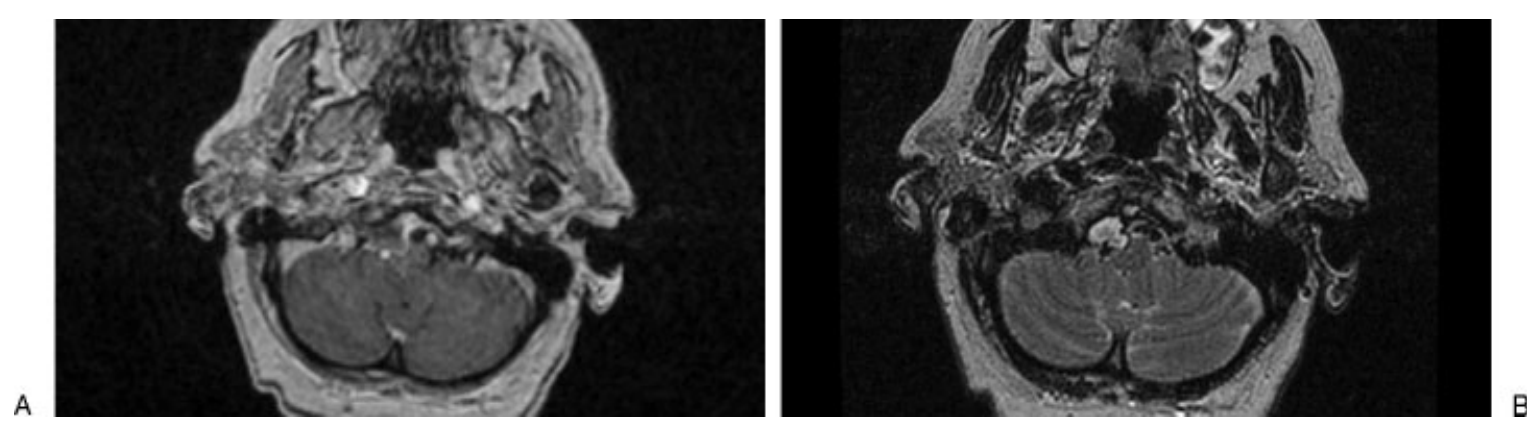

Figure 1 Preoperative radiographic imaging. (A) Axial magnetic resonance imaging (MRI) of the brain, T1 postcontrast, revealing minimal enhancement of a right-sided clival hypointense lesion with compression of the medulla oblongata. (B) Axial $\mathrm{MRI}$ of the brain, T2, revealing same lesion demonstrating hyperintense features.

lower part of the clivus in the upper aspect of the odontoid process of the cervical C2. The presumptive diagnosis was a clival chordoma, and we performed an endoscopic transnasal transclival resection of the mass, which was shown with pathological review to be consistent with a degenerative pannus. Unique features of the case are presented, along with a discussion of the skull base approach and a review of the literature.

\section{CASE REPORT}

A 75-year-old woman with a history of osteoarthritis, hypertension, and pulmonary embolism and a remote history of smoking presented with complaints of loss of balance and increasing falls over the course of 1 year. The patient did not have a known history of rheumatoid arthritis. Her symptoms began after she experienced a transient ischemic attack during which she had a sudden onset of drooling and slurred speech that improved over a few hours. Yet over the course of the next few months, her symptoms gradually returned and became persistent. She eventually noticed disfigurement of her tongue.

On examination, the patient had full motor function in all myotomes and normal deep tendon reflexes $(2+)$ in both the upper and lower extremities. Her cranial nerve exam revealed a right hypoglossal nerve palsy associated with significant tongue atrophy, but the other cranial nerves were noted to be intact. Magnetic resonance imaging (MRI) of the brain revealed a heterogenous lesion of the right inferior clivus, which was interpreted as a clival chordoma by an outside hospital. MRI further demonstrated extra-axial compression of the medulla oblongata with associated T2 hyperintensity of the effaced portions of the brain stem (Fig. 1). A CT scan confirmed the presence of an extra-axial lesion at the craniovertebral junction and also highlighted degenerative changes at the atlantoaxial and atlantooccipital joints (Fig. 2). Based on the available imaging, our differential diagnosis included chordoma, cystic meningioma, chondrosarcoma, and metastatic disease, but because of the radiographic appearance and associated $\mathrm{C} 1-\mathrm{C} 2$ disease, we considered rheumatoid or another degenerative process high on our list as well.

\section{SURGERY}

Prior to surgery, fine-cut MRI and CT scans of the brain were obtained for neuronavigation. On the day of

A
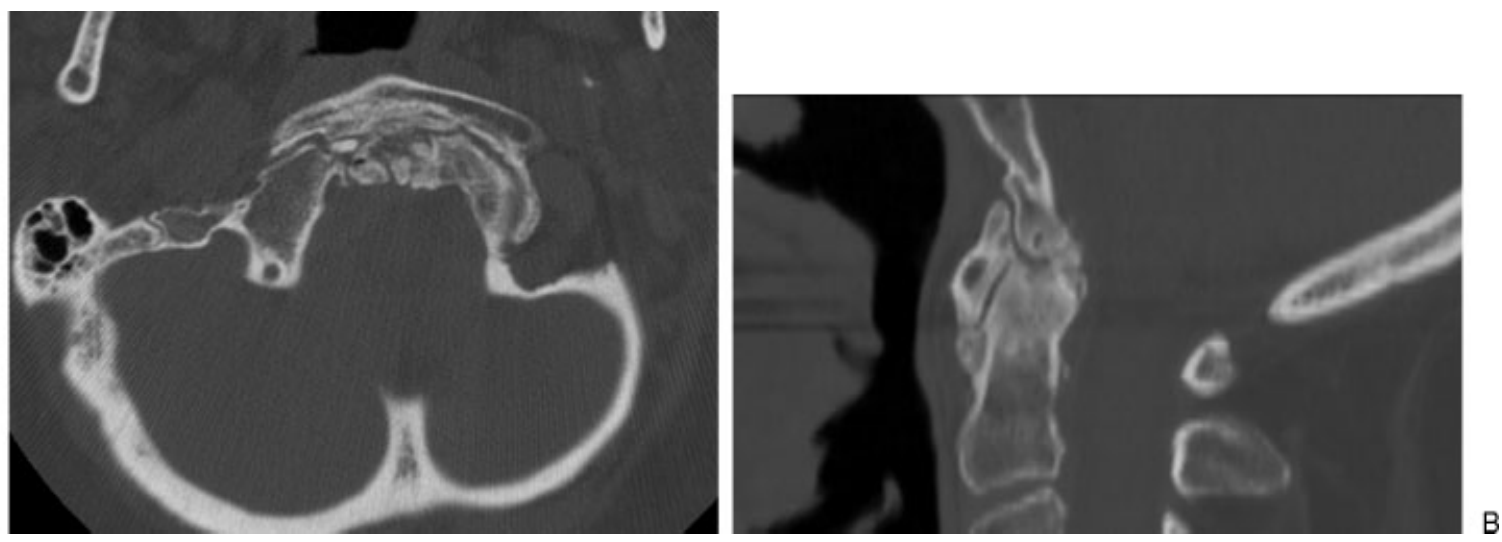

Figure 2 (A) Axial computed tomography (CT) scan of the brain, showing extensive degenerative changes at cervical $\mathrm{C} 1$ and $\mathrm{C} 2$ and the atlantooccipital joint. (B) Reconstruction CT scan of the cervical spine revealing degenerative changes at the odontoid process as well as the lower clivus and the $\mathrm{C} 1$ ring. 
surgery, the patient was orotracheally intubated, and the lower cranial nerves monitored. Using a zero-degree endoscope, the patient underwent a transnasal-transclival resection of the lesion, which we felt was most likely extradural. A left-sided septal mucosal flap (with attached vascular pedicle) was harvested and stored in the nasopharynx for use at the end of the case in the event the lesion was found to be intradural, a CSF leak was encountered, or an extensive reconstruction was necessary. The right middle turbinate was resected and a posterior septotomy performed to give binarial access to the region of the clivus.

Without entering the sphenoid sinus, and with our trajectory determined by the use of anatomic landmarks as well as image guidance, a paramedian incision in the posterior pharynx was made and the incision extended into the superior pharyngeal musculature. The small linear incision allowed for exposure of the clival bone at a depth of $\sim 1 \mathrm{~cm}$ from the mucosal surface. The superior aspect of the anterior ring of $\mathrm{C} 1$ was palpated and exposed. A drill was used to create a bony window in the right side of the clivus, revealing the lesion. The heterogeneous fibrotic mass was removed with sharp and blunt dissection techniques until the underlying dura was exposed and all extradural effacement of the posterior fossa had been alleviated. The lesion was removed circumferentially with no associated dural violation or cerebrospinal fluid (CSF) leak. The mucosal flap was then used to close the pharyngeal mucosal deficit with "tacking" of the flap in place accomplished with DuraSeal (Confluent Surgical, Waltham, MA). The patient tolerated the procedure well and emerged from anesthesia without complication. Estimated blood loss for the procedure was $200 \mathrm{~mL}$.

The patient had no new neurological deficits after the operation. There was no evidence of a CSF leak. She maintained a normal range of motion in her neck, and flexion/extension cervical spine $\mathrm{X}$-rays demonstrated no instability. A postoperative MRI scan of the brain demonstrated complete resolution of the clival lesion (Fig. 3). The patient left the hospital on postoperative day 4. The pathology of the lesion revealed benign fibrous capsule with fragments of necrotic material con- sistent with degenerative disease, which was not associated with malignancy. The patient underwent nasal suctioning and debridement at 2 weeks postoperation and continued twice daily nasal flushes. Nasal morbidity was otherwise minimal. At 7 weeks postoperatively, the patient had regained some bulk on the right side of her tongue, but it was still paretic.

\section{DISCUSSION}

The endoscope has become an increasingly popular tool for approaching the sella and resecting pituitary tumors. ${ }^{10}$ Endoscopic techniques have also been applied to lesions of the clivus, petrous bone, cavernous sinus, and infratemporal fossa. ${ }^{3-5,11}$ Endoscopy allows for excellent visualization of key structures and pathologies in the clival region, ${ }^{10,12-14}$ while avoiding the significant morbidity that can be associated with traditional skull base procedures. These approaches often involve a large scalp incision, extensive bone work, and brain retraction. They can have the potential for morbidity and extended hospital stays and may involve cranial nerve injury. Traditional skull base approaches to the clivus have included extended subfrontal-transnasal transethmoidal, ${ }^{15}$ transoral, ${ }^{12,16}$ transmandibular circumglossal, ${ }^{17}$ pterional, frontotemporal orbitozygomatic, ${ }^{18}$ Kawase's approach, ${ }^{19}$ subtemporal, ${ }^{20}$ translabyrinthine, posterior petrosectomy, retrosigmoid, and far lateral. ${ }^{21}$ Even CTguided biopsy is not without risk due to its indirect approach, or the potential for ambiguous results due to small biopsy sample size.

Endoscopic approaches have been shown to have low risks of complication and have evolved into a viable alternative to craniotomy and CT-guided biopsy when addressing lesions of the clivus. ${ }^{2,12,22}$ Although endoscopic approaches may have lower overall risks than other procedures, they are associated with a high risk of CSF leak. ${ }^{2-5}$ Unintended and intentional durotomies can be repaired intraoperatively by using a mucosal flap or fat graft, but especially in the case of the mucosal flap; the surgeon(s) must plan ahead for these contingencies. ${ }^{12}$ Other drawbacks of the endoscopic approach include the lack of three-dimensional optics (although this is
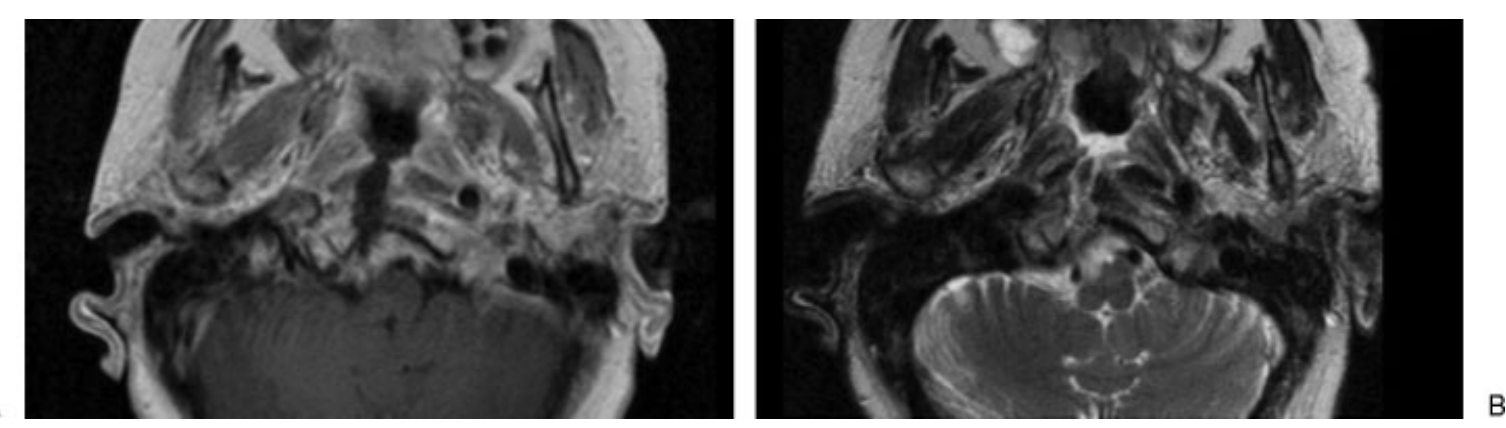

Figure 3 Postoperative magnetic resonance imaging (MRI). (A) Axial MRI of the brain, T1 with contrast, shows complete resection of tumor. (B) Axial MRI of the brain, T2, highlights decompression of the brain stem. 
changing), a technical learning curve for endoscopic techniques, diminished ability to control bleeding, and difficulty in achieving en bloc resection. ${ }^{3}$

Transoral endoscopic approaches allow for a wide working area and contain familiar anatomy. ${ }^{23}$ Additional advantages include an approach through the avascular midline raphe as well as decreased angulation of the brain stem due to an extension of the head and neck in the operating room as opposed to flexion. ${ }^{24}$ Disadvantages include the need for nasogastric tube (NGT) use to prevent food getting stuck in the healing pharynx. ${ }^{25}$ Similarly, progression to normal diet can be slow with up to 5 days needed for NGT, then several days of clear liquid diet, then attempts at a full diet. In our case, our patient did not require an NGT; she tolerated a clear liquid diet on postoperative day 1 , and by postoperative day 3, she was tolerating a general diet. Additional disadvantages of the transoral approach include possible need for soft palate splitting, posterior oropharyngeal incision, compression to the tongue from the required retraction, airway swelling, upper airway obstruction, velopharyngeal insufficiency, damage to teeth by retractors, dysphonia, and a deep surgical corridor. ${ }^{23} \mathrm{We}$ avoided these complications by employing a transnasal approach. The transnasal approach is not risk free, however. Possible morbidities include nasal crusting, epistaxis, and infections. ${ }^{3}$

Both benign and malignant lesions can invade the clivus. Common lesions include chordomas, chondrosarcomas, meningiomas, pituitary adenomas, undifferentiated carcinomas, adenoid cystic cancers, fibrous dysplasia, and mucoceles. ${ }^{2,3,12}$ Due to the assortment of pathology in this anatomic region, definitive radiological diagnosis can be difficult. Imaging studies can include plain radiographs, CT scans, and MRI with or without magnetic resonance spectroscopy. Osseous erosion and anatomy can be best evaluated using CT scan, ${ }^{7}$ whereas MRI best highlights tumor characteristics, soft tissue anatomy, and neural involvement. In rheumatoid patients, typical MRI characteristics include erosion of the dens or atlas, cord compression, brain stem compression, and the presence of moderate to severe pannus formation. ${ }^{26}$ Indications for surgery include the need for a tissue diagnosis, intractable pain, neurological involvement, and occipitocervical instability. ${ }^{7}$

The clivus is one of the most common areas for chordomas, which represent malignant remnants of the embryonic notochord. ${ }^{12}$ Clival chordomas grow slowly and are locally damaging. Recurrence of chordomas is very common and depends on the initial extent of involvement and the degree of resection. ${ }^{27}$ Total resection is difficult, leading to a high rate of recurrence. Recurrent chordomas are typically more aggressive and difficult to control than primary ones. ${ }^{28,29}$ The 5 -year survival rate for all comers is around $65 \%{ }^{30,31}$; however, the survival rate improves to $75 \%$ if the patient is diagnosed before age $40 .^{32}$ On the other hand, the overall recurrence rate is high, ranging from 31 to $54 \%{ }^{27,33}$ With increasing utilization of the endoscope, it will be interesting to see if recurrence rates are affected. This approach, with its advantages of bright illumination and close visualization of tumor and surrounding structures alike, may lead to more complete resections during primary chordoma surgery. ${ }^{13,14}$ In addition, the endoscopic route offers an alternate "virgin" approach for patients with recurrence who have previously undergone craniotomy in whom second-stage surgery is warranted. ${ }^{13}$

Chondrosarcomas appear similar to chordomas and can also invade the clivus, but can be differentiated pathologically by immunohistochemistry. ${ }^{34}$ These lesions are believed to be derived from either embryonal cartilage or from altered fibroblasts. ${ }^{12,35}$ Diagnostic differentiation is important because chondrosarcomas have better survival and recurrence rates then chordomas. ${ }^{12}$ Meningiomas can commonly grow in the clival region, and pituitary macroadenomas can extend into the clivus from the floor of the sella. ${ }^{36}$

Rheumatoid disease, by contrast, most often occurs at or below the craniovertebral junction, frequently behind the odontoid process. ${ }^{9}$ Rheumatoid arthritis is an autoimmune process involving synovial joints and can lead to atlantoaxial instability and subluxation, cranial settling, and basilar invagination. ${ }^{8} \mathrm{~A}$ common feature of rheumatoid disease is pannus formation via an inflammatory and granulation reaction. This is a common cause of spinal cord or brain stem compression when it occurs retro-odontoid or at the atlantooccipital junction. ${ }^{9,37,38}$ In our case, the compression was more rostral, and the corresponding treatment had to be different. In many cases of retroodontoid rheumatoid pannus formation, especially those associated with $\mathrm{C} 1-\mathrm{C} 2$ instability, posterior stabilization of the atlantoaxial joint via $\mathrm{C} 1-\mathrm{C} 2$ or occipitocervical fusion allows for resolution of the pannus and associated mass effect. In our case, such a treatment strategy would not have been likely to succeed.

\section{CONCLUSION}

Degenerative pannus should be included in the differential diagnosis of lower clival extradural lesions. The endoscopic transnasal trasclival corridor should be considered for resection of such lesions, or even intradural lesions (as a surgeon's comfort and ability to perform dural reconstruction progresses), as an alternative to larger and more morbid traditional skull base approaches. The endoscopic approach provides the surgeon with excellent illumination and visualization of these lesions while allowing for resection and accurate pathological diagnosis. The minimally invasive nature of the endoscopic approach allows for a potentially shorter recovery period, and the direct trajectory associated 
with the transclival approach allows for avoidance of injury to neural structures.

\section{REFERENCES}

1. Rhoton AL, Congress of Neurological Surgeons. Cranial Anatomy and Surgical Approaches. Hagerstown, MD: Lippincott Williams \& Wilkins; 2003

2. Kelley TF, Stankiewicz JA, Chow JM, Origitano TC. Endoscopic transsphenoidal biopsy of the sphenoid and clival mass. Am J Rhinol 1999;13:17-21

3. Solares CA, Fakhri S, Batra PS, Lee J, Lanza DC. Transnasal endoscopic resection of lesions of the clivus: a preliminary report. Laryngoscope 2005;115:1917-1922

4. Laws ER Jr. Transsphenoidal surgery for tumors of the clivus. Otolaryngol Head Neck Surg 1984;92:100-101

5. Maira G, Pallini R, Anile C, et al. Surgical treatment of clival chordomas: the transsphenoidal approach revisited. J Neurosurg 1996;85:784-792

6. Kassam AB, Gardner P, Snyderman C, Mintz A, Carrau R. Expanded endonasal approach: fully endoscopic, completely transnasal approach to the middle third of the clivus, petrous bone, middle cranial fossa, and infratemporal fossa. Neurosurg Focus 2005;19:E6

7. Shen FH, Samartzis D, Jenis LG, An HS. Rheumatoid arthritis: evaluation and surgical management of the cervical spine. Spine J 2004;4:689-700

8. Nguyen HV, Ludwig SC, Silber J, et al. Rheumatoid arthritis of the cervical spine. Spine J 2004;4:329-334

9. de Almeida JR, Zanation AM, Snyderman $\mathrm{CH}$, et al. Defining the nasopalatine line: the limit for endonasal surgery of the spine. Laryngoscope 2009;119:239-244

10. Jho HD. Endoscopic pituitary surgery. Pituitary 1999;2: 139-154

11. de Divitiis E, Cappabianca P, Cavallo LM. Endoscopic transsphenoidal approach: adaptability of the procedure to different sellar lesions. Neurosurgery 2002;51:699-705; discussion 705-707

12. Frank G, Sciarretta V, Calbucci F, et al. The endoscopic transnasal transsphenoidal approach for the treatment of cranial base chordomas and chondrosarcomas. Neurosurgery 2006; 59(Suppl 1): ONS50-ONS57; discussion ONS50-ONS57

13. Stippler M, Gardner PA, Snyderman CH, et al. Endoscopic endonasal approach for clival chordomas. Neurosurgery 2009;64:268-277; discussion 277-278

14. Dehdashti AR, Karabatsou K, Ganna A, Witterick I, Gentili F. Expanded endoscopic endonasal approach for treatment of clival chordomas: early results in 12 patients. Neurosurgery 2008;63:299-307; discussion 307-309

15. Sekhar LN, Nanda A, Sen CN, Snyderman CN, Janecka IP. The extended frontal approach to tumors of the anterior, middle, and posterior skull base. J Neurosurg 1992;76: 198-206

16. Crockard HA, Sen CN. The transoral approach for the management of intradural lesions at the craniovertebral junction: review of 7 cases. Neurosurgery 1991;28:88-97; discussion 97-98

17. Price JC. The midfacial degloving approach to the central skull-base. Ear Nose Throat J 1986;65:174-180

18. Froelich S, Aziz KA, Levine NB, et al. Extension of the onepiece orbitozygomatic frontotemporal approach to the glenoid fossa: cadaveric study. Neurosurgery 2008;62(Suppl 2): ONS312-ONS316; discussion ONS316-ONS317

19. Aziz KM, van Loveren HR, Tew JM Jr, Chicoine MR. The Kawase approach to retrosellar and upper clival basilar aneurysms. Neurosurgery 1999;44:1225-1234; discussion 1234-1236

20. Tanriover N, Sanus GZ, Ulu MO, et al. Middle fossa approach: microsurgical anatomy and surgical technique from the neurosurgical perspective. Surg Neurol 2009;71:586-596; discussion 596

21. Lanzino G, Dumont AS, Lopes MB, Laws ER Jr. Skull base chordomas: overview of disease, management options, and outcome. Neurosurg Focus 2001;10:E12

22. Frempong-Boadu AK, Faunce WA, Fessler RG. Endoscopically assisted transoral-transpharyngeal approach to the craniovertebral junction. Neurosurgery 2002;51(Suppl): S60-S66

23. Baird CJ, Conway JE, Sciubba DM, Prevedello DM, Quiñones-Hinojosa A, Kassam AB. Radiographic and anatomic basis of endoscopic anterior craniocervical decompression: a comparison of endonasal, transoral, and transcervical approaches. Neurosurgery 2009;65(Suppl): 158-163; discussion 63-64

24. Menezes AH. Surgical approaches: postoperative care and complications "transoral-transpalatopharyngeal approach to the craniocervical junction”. Childs Nerv Syst 2008;24:11871193

25. Yang SY, Gao YZ. Clinical results of the transoral operation for lesions of the craniovertebral junction and its abnormalities. Surg Neurol 1999;51:16-20

26. Reijnierse M, Dijkmans BA, Hansen B, et al. Neurologic dysfunction in patients with rheumatoid arthritis of the cervical spine. Predictive value of clinical, radiographic and MR imaging parameters. Eur Radiol 2001;11:467-473

27. Pallini R, Maira G, Pierconti F, et al. Chordoma of the skull base: predictors of tumor recurrence. J Neurosurg 2003;98: 812-822

28. Park L, Delaney TF, Liebsch NJ, et al. Sacral chordomas: impact of high-dose proton/photon-beam radiation therapy combined with or without surgery for primary versus recurrent tumor. Int J Radiat Oncol Biol Phys 2006;65: 1514-1521

29. Hug EB, Loredo LN, Slater JD, et al. Proton radiation therapy for chordomas and chondrosarcomas of the skull base. J Neurosurg 1999;91:432-439

30. Mendenhall WM, Mendenhall CM, Lewis SB, Villaret DB, Mendenhall NP. Skull base chordoma. Head Neck 2005; 27:159-165

31. Colli B, Al-Mefty O. Chordomas of the craniocervical junction: follow-up review and prognostic factors. J Neurosurg 2001;95:933-943

32. Forsyth PA, Cascino TL, Shaw EG, et al. Intracranial chordomas: a clinicopathological and prognostic study of 51 cases. J Neurosurg 1993;78:741-747

33. Fagundes MA, Hug EB, Liebsch NJ, Daly W, Efird J, Munzenrider JE. Radiation therapy for chordomas of the base of skull and cervical spine: patterns of failure and outcome after relapse. Int J Radiat Oncol Biol Phys 1995;33: 579-584

34. Rosenberg AE, Nielsen GP, Keel SB, et al. Chondrosarcoma of the base of the skull: a clinicopathologic study of 200 cases with emphasis on its distinction from chordoma. Am J Surg Pathol 1999;23:1370-1378 
35. Korten AG, ter Berg HJ, Spincemaille GH, van der Laan RT, Van de Wel AM. Intracranial chondrosarcoma: review of the literature and report of 15 cases. J Neurol Neurosurg Psychiatry 1998;65:88-92

36. Goel A, Phalke U, Cacciola F, Muzumdar DP. Giant pituitary adenoma invading the clivus. Neurol India 2005;53: 105-107
37. Quarta L, Corrado A, Melillo N, et al. Cervical myelopathy caused by periodontoid synovial pannus in a patient with psoriatic arthritis: a case report. Clin Rheumatol 2007;26: 1380-1382

38. Chugh R, Tawbi H, Lucas DR, Biermann JS, Schuetze SM, Baker LH. Chordoma: the nonsarcoma primary bone tumor. Oncologist 2007;12:1344-1350 